\title{
Effect of powdered shells treatment of the snail Megalobulimus lopesi on wounds of diabetic rats ${ }^{1}$
}

Paulo Henrique Muleta Andrade', Luciane Canderolo Portugal", Eric Schmidt Rondon"', Monica Cristina Toffoli Kadriv ${ }^{\prime v}$, Maria de Fátima Cepa Matos ${ }^{\vee}$

'Fellow PhD degree, Postgraduate Program in Health and Development of the Central West Region, Universidade Federal do Mato Grosso do Sul (UFMS), Campo Grande-MS, Brazil. Acquisition, analysis and interpretation of data; manuscript writing.

"PhD, Department of Morphophysiology, UFMS, Campo Grande-MS, Brazil. Histopathological examinations, manuscript preparation, critical revision.

I'PhD, Department of Veterinary Medicine, UFMS, Campo Grande-MS, Brazil. Acquisition, analysis and interpretation of data; manuscript preparation; critical revision.

IVPhD, Center for Biological and Health Sciences (CCBS), UFMS, Campo Grande-MS, Brazil. Manuscript preparation, critical revision.

${ }^{V}$ PhD, Faculty of Pharmaceutical Sciences, Food and Nutrition, UFMS, Campo Grande-MS, Brazil. Acquisition, analysis and interpretation of data; manuscript preparation, critical revision.

\section{Abstract}

Purpose: To analyzed the healing effect of the powdered shell of the Megalobulimus lopesi snail on wounds of diabetic rats, since in non-diabetic rats the powdered shell presented healing potential.

Methods: Seventy-two Wistar rats (Rattus norvegicus albinus) were divided into three groups: Control group (GC.diab), no therapeutic intervention on the wound; Vehicle's Control group, topical via, in diabetic rats (GCvt.diab): Powder Shell Group (PC) applied topically (GPCvt.diab): Experimental group was administered topically shortly after wound dressing and once a day during the experimental period (3, 7, 14 and 21 days) the composition containing the powdered shell of the snail. The following variables related to the healing potential were analyzed: macroscopic one, where the capacity of reduction of the wound area was evaluated; histological analysis in $\mathrm{HE}$, angiogenic activity, morphometric analysis (re-epithelization), leukocyte inflammatory infiltrate; leukocyte count and also differentiation in peripheral blood.

Results: The topical application in wounds of diabetic rats presented healing activity, accelerating wound closure, stimulating angiogenesis and being pro-inflammatory in the early and anti-inflammatory stages in the final times of the healing process.

Conclusion: The topical administration of the powdered shell on wounds of diabetic patients becomes a therapeutic option of low cost, with ease in the administration and access as well. Key words: Megalobulimus lopesi. Wound Healing. Diabetes Mellitus. Rats. 


\section{Introduction}

It is estimated that approximately $10 \%$ of the world population is diabetic, being considered the fourth most non-transmissible lethal disease in the world, leading to 1.3 million deaths per year due to its complications ${ }^{1}$.

Hyperglycaemia is the main characteristic of Diabetes Mellitus (DM). In physiological conditions the regulation of glycemia is maintained by means of a delicate balance between insulin secretion and insulin sensitivity. Thus, an initial sign of glucose intolerance and / or a decrease in insulin sensitivity in peripheral tissues results in the compensatory increase of insulin secretion to maintain normoglycemia ${ }^{2}$.

Among the several complications associated with diabetes, the most common is the impairment of wound healing and the occurrence of wounds, especially in the feet (diabetic foot), consequently diabetic individuals have a risk of amputation 15 times higher, when compared to non-diabetic ${ }^{2}$. Corroborating with these data, World Health Organization (WHO) reported that 150 million people developed wounds due to DM, and the estimate for 2025 is twice as high ${ }^{1}$.

In cutaneous wounds in the diabetic individual, wound healing can be blocked at different stages, losing the synchrony required for normal and rapid regeneration ${ }^{3}$. The slow regeneration may have as intrinsic causes: hyperglycemia, neuropathy, macro and micro angiopathies, as extrinsic: infections, callus formation and excessive pressure at the wound site (caused by inadequate shoes) ${ }^{4}$.

In addition, the healing activity of the $M$. lopesi shell in rat wounds was demonstrated, where, before 21 days of healing, wound area was closed, associated with an increase in the number of inflammatory cells and accentuated angiogenesis ${ }^{5}$. Therefore, in this study we analyzed the effect of treatment of cutaneous wounds in diabetic rats with the powdered shell of snail M. Lopesi.

\section{Methods}

\section{Shell collection and ointment preparation}

The snails were purchased from a private estate specializing in the cultivation and rearing of mollusks and were transported alive to the laboratory. After euthanasia, the shell was separated from the remaining tissues. After the shell was insulated, it was washed with distilled water and then immersed in liquid nitrogen to inactivate possible enzymes and maintain the organic and protein structures. Once Freeze-dried, the shell was broken and pulverized in a Marconi ${ }^{\circ}$ MA701 / 21 ball mill ${ }^{5}$.

For topical administration, an ointment was prepared using Lanete ${ }^{\bullet}$ watersoluble anionic vehicle (Dermavita Indústria Cosmética), a 50\%- concentration added of the Powdered shellwas added just after. The ointment was prepared at the Laboratory of Pharmaceutical Technology.

\section{In vivo experimentation}

All experiments and procedures were approved by the Animal Research Ethics Committee, Universidade Federal do Mato Grosso do Sul under permit number 630/2014.

A total of 72 rats (Rattus norvegicus albinus) of the Wistar lineage were used, males aged 45 days, weighing $145 \pm 10$ grams, coming from the Central Biomedical Unit of UFMS. All animals were housed in a temperature controlled room at $23^{\circ} \mathrm{C}$, one animal per plastic cage and kept in an alternating 12-hour lightdark cycle. Food and water were supplied ad libitum.

\section{Wound dressing}

All rats were submitted to the surgical 
procedure to make the skin wound in the dorsal region.

The anesthetic procedure was performed by intramuscular injection of Ketamine Hydrochloride $(25 \mathrm{mg} / \mathrm{kg}$ ) and Xilasine Hydrochloride (5 $\mathrm{mg} / \mathrm{kg})$, with a maximum volume of $0.5 \mathrm{~mL}$. All surgical procedures were developed following an antisepsis protocol. After the dorsal region shaving, all animals were underwent to a cutaneous tissue excision of $0.6 \mathrm{~cm}$ diameter using a Keller Punch, performed in the center of the shaving area, exposing the muscular fascia of the paravertebral musculature. Finally, hemostasis was performed with digital compression, by using sterile gauze.

\section{Diabetic rats}

The induction of diabetes in the animals was performed by intraperitoneal injection of alloxan solution (5,6-dioxy uracil monohydrate) in a single dose of $150 \mathrm{mg} / \mathrm{kg}$ body weight administered after a previous 18-hour fast. In the first 24 hours of induction, the animals will receive a $10 \%$ glucose solution to avoid animal mortality due to the massive release of insulin after destruction of the pancreatic beta cells.

A week after the application of the referred drug, the condition was characterized by polyuria, polydipsia and capillary glycemia over $200 \mathrm{mg} / \mathrm{dL}$. For the capillary glycemia test, a surgical styled incision was made in the distal part of the animal's tail with sufficient opening to collect a drop of blood for the glycemic test.

Once the diabetes mellitus condition was confirmed, the animals ( $n=72)(n=6$ per group) were submitted to the surgical procedure as previously described, and were then randomly assigned to three groups:

- Control group (GC.diab), no therapeutic intervention on the wound;

- Vehicle control group, topical administration, in diabetic rats (GCvt.diab): Topical vehicle control group, was administered topically shortly after wound dressing and once a day during the trial period the vehicle;

- Powdered Shell Group (PC), applied topically (GPCvt.diab): Experimental group, it was administered topically shortly after wound dressing and once day during the experimental period the composition containing the powdered shell of the snail;

All treatments started soon after the surgical wound was made and were kept once a day during the experimental period.

The animals ( $\mathrm{n}=6$ per group) were euthanized 3, 7, 14 and 21 days after wound dressing. For this purpose, they were anesthetized for the collection of the material for macroscopic analysis, peripheral blood for leukocyte count and removal of the tissue fragment for histological analysis. They were placed, then, in an open $\mathrm{CO}_{2}$ chamber for 60 seconds, remaining in it until the absence of vital signs (3 to 5 minutes).

\section{Macroscopic analysis}

Wounds were analyzed macroscopically for the ability to promote contraction of the lesion.

The distances of the wound edges in the longitudinal and transverse directions were measured with a digital caliper (Mitutoyo ${ }^{\circ}$-11237). The arithmetic mean of these two measures was converted into a percentage value and used in the statistical tests. Thus, immediately after wound healing (day zero or $\mathrm{T}=0$ ), the percentage value of $100 \%$ was considered, i.e. fully open wound. On the subsequent days (days 3, 7, 14 and 21, or respectively $T=3, T=7, T=14$ and $T=21$ ) the arithmetic means obtained were converted into percentages of open wound quantifying thus, the closing of the surgical wound.

The proportion of lesion area at the initial time of the wound was evaluated in relation to the posterior days $(3,7,14$ and 21 days). For the statistical analysis, the values 
obtained in both directions were summed and divided by two to obtain an average value of proportion.

\section{Histological analysis}

The tissue fragment removed from the rat with the surgical wound was preserved in $10 \%$ formalin solution and processed according to the parameters for evaluation under light microscopy. Included in paraffin, crosssections were made at the wound site with a thickness of $5 \mu \mathrm{m}$, the slides were stained using the hematoxylin-eosin (HE) method. They were analyzed and photographed under a microscope (Jenaval-Zeiss) with a coupled camera (Leica DFC425).

In the microscopy analysis the following parameters were evaluated: angiogenic activity, morphometric analysis, leukocyte inflammatory infiltrate.

\section{Angiogenic activity}

Blood vessel count was performed in 10 fields per slide at 7, 14 and 21 days using light microscopy on an objective lens with 20x magnification. Data were expressed as the mean in each lesion.

\section{Morphometric analysis}

The thickness of the re-epithelial tissue was measured by photomicrographs obtained from the videomicroscopy system, and the thickness was evaluated from dermal-junction to the outermost portion of the granular layer. Three random fields per animal were analyzed using the objective lens at 20x magnification, with three measurements in each field. Data were expressed as the mean in each lesion.

\section{Inflammatory leukocyte infiltrate}

The number of cells of the leukocyte inflammatory infiltrate were analyzed, expressed in number of cells per $\mu \mathrm{m}^{2}(\mathrm{QA})^{6}$. Lesion tissue sections stained with $\mathrm{HE}$ were analyzed in 10 random fields per animal (3305 $\mu \mathrm{m}^{2}$ ) with the $\mathrm{x} 40$ objective lens. Only the cells present within the field were counted, excluding cells that touched the edge of the field. Data were represented as the mean of each lesion.

\section{Leukocyte count}

For the total leukocyte count, $20 \mu \mathrm{L}$ of blood from the orbicularis artery was removed and it was added to them $380 \mu \mathrm{L}$ of Turk Fluid (1:20). For the differential count, another aliquot was used to make a smear on a slide and stained in $\mathrm{Hema}^{\circ}$. Results were expressed as number of cells per $\mathrm{mm}^{3}$.

\section{Statistical analysis}

All data were expressed by the means \pm standard deviation of the means (means \pm SEM). The data analysis was performed comparing the mean of the groups, for the healing times and between the groups (two-way ANOVA). For data on wound area contraction, total leukocyte count, angiogenic activity, leukocyte inflammatory infiltrate and morphometric analysis, Tukey's post hoc test was applied. Values at $\mathrm{P}<0.05$ were considered statistically significant. The SigmaStat version 3.5 program was used for the statistical analysis.

\section{Results}

All animals presented clean wounds without apparent changes. Besides, no signs of self-injury or bite were observed at the wound site. The management was the same for all animals in all groups.

All the animals used in the experiments had a capillary glycemic rate above $200 \mathrm{mg} / \mathrm{dL}$ (Figure 1), as well as the signs of polyuria and polydipsia, which characterize diabetes. 


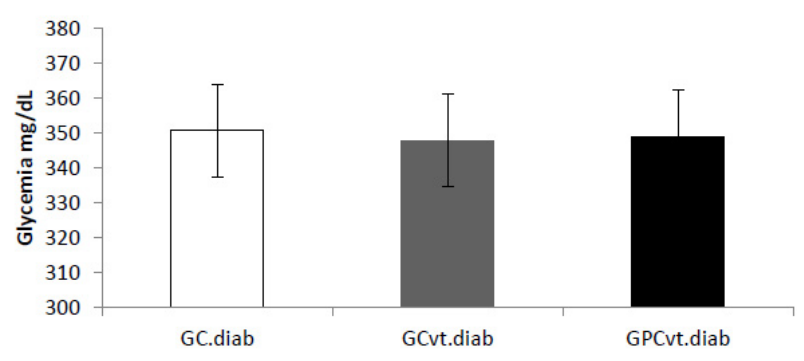

Figure 1 - Capillary glycemia $\mathrm{mg} / \mathrm{dL}$. Data are presented as mean \pm standard deviation of the mean (SEM) ( $n=24)$. Diabetic Group Control (GC. diab), Topic Vehicle Control Group (GCvt.diab) and Experimental Group of Powdered shell (GPCvt. diab).

\section{Wound area}

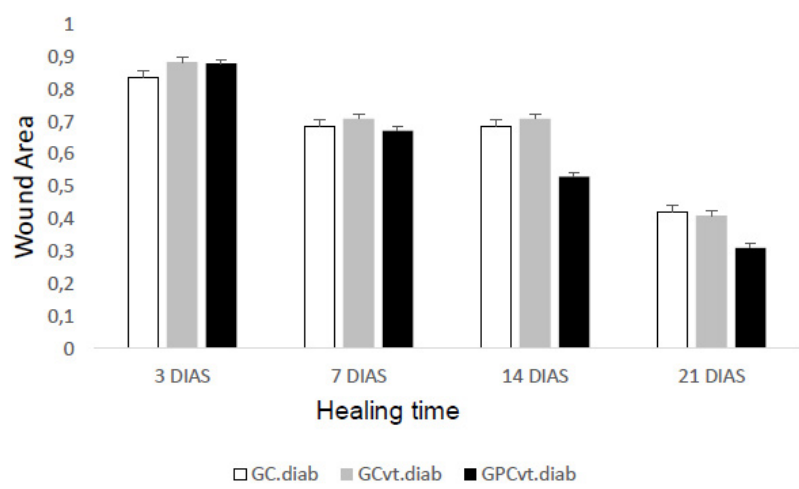

Figure 2 - Relation of the wound area in the different times of healing. Data are presented as mean \pm standard deviation of the mean (SEM) ( $n=$ 6). Diabetic Control Group (GC.diab), Topic Vehicle Control Group (GCvt.diab) and Experimental Group of Powdered shell (GPCvt.diab).

In the general analysis of the results concerning the proportion of the wound area in relation to the initial postoperative time, there was an effect of the group, the time of the analysis and the interaction between these factors (two-way ANOVA test of repetitive measures, $p<0.001$ ). In the analysis per groups along different healing times, a significant reduction in the proportion of the wound area was observed, in relation to the initial time
(Tukey's post-test, $p<0.05$ ). For the groups GC.diab and GCvt.diab there was no significant difference between the 7 and 14 days ( $p>0.05$ ) times. This result that was not observed for the GCPct.diab, that presented progressive reduction of the wound area $(p<0.05)$ at all healing times. In the comparison among the experimental groups at each analysis time, it was evidenced that the proportion of the wound area for the GCPct.diab group at 14 and 21 days was lower in relation to the GC.diab and GCvt.diab groups (post-test Tukey, $p<0.001$ ), and there was no significant difference among the groups in 3 and 7 days of healing.

\section{Angiogenic activity}

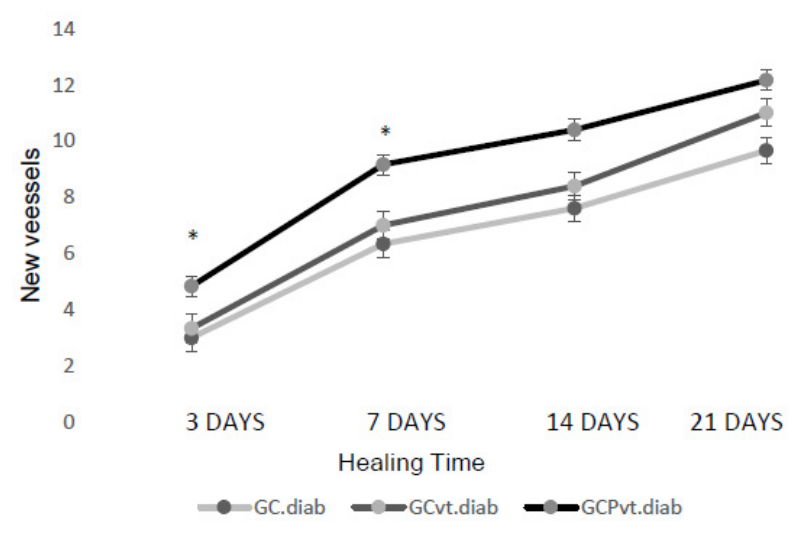

Figure 3 - Increase in the number of new vessels per analyzed field, at the lesion site, in each experimental group. Data represent the mean \pm standard deviation of the mean (SEM) $(n=6)$. Group Diabetic Control (GC.diab), Topic Vehicle Control Group (GCvt.diab) and Experimental Group of Powdered Shell (GPCvt.diab). ${ }^{*} P<0.001$.

In the general analysis of the results regarding the increase in the number of new vessels at the lesion site, there was an effect of the experimental group, the time of analysis and interaction between these factors (twoway ANOVA test of repetitive measures, $p$ $<0.001)$. 
In the group analysis at different healing times, there was a significant increase in the number of new vessels at the lesion site (Tukey's post-test, $p<0.05$ ), where all groups presented progressive increase at the lesion site.

At 3 and 7 days of healing, GCPvt.diab presented a higher number of new vessels in relation to the other groups, but in 14 and 21 days of healing there was no significant difference between the three experimental groups (Tukey's post-test, $p<0.05$ ).

\section{Morphometric analysis of new epithelium} thickness

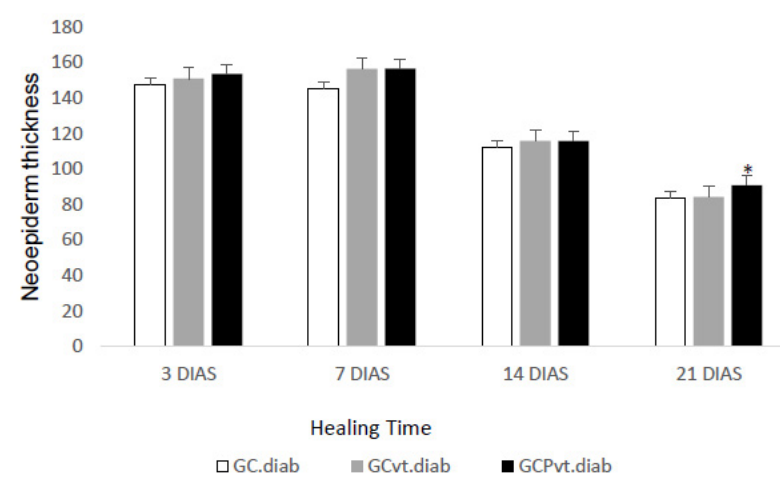

Figure 4 - New epithelium thickness for the groups on analysis times 3, 7, 14 and 21 days. Data are presented as the mean \pm standard deviation of the mean (SEM) $(n=6)$. Diabetic Control Group (GC. diab), Topic Vehicle Control Group (GCvt.diab) and Experimental Group of Powdered shell (GPCvt. diab). ${ }^{*} \mathrm{P}<0.001$.

In the general analysis of the results regarding the morphometric analysis of the new epithelium, there was an effect of the moment of analysis (ANOVA test of two replicate measures, $\mathrm{p}<0.001)$.

In the group analysis in different healing times, there was a significant reduction in new epithelium thickness after 14 days of healing (Tukey post-test, $\mathrm{p}<0.05$ ).

On the 21th day of healing, GCPvt.diab had a greater new epithelium thickness when compared to the other groups (Tukey's posttest, $\mathrm{p}<0.05$ ).

\section{Leukocytic inflammatory infiltration}

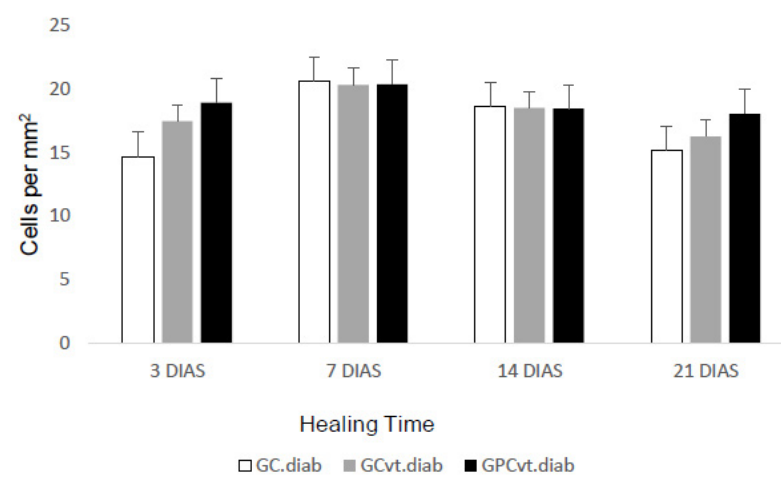

Figure $\mathbf{5}$ - Leukocyte inflammatory infiltrate per field analyzed, at the site of the lesion, in each experimental group in analysis times 3, 7, 14 and 21 days. Data are presented as the mean \pm standard deviation of the mean (SEM) $(n=6)$. Diabetic Control Group (GC.diab), Topic Vehicle Control Group (GCvt.diab) and Experimental Group of powdered shell (GPCvt.diab).

In the general analysis of the results concerning the inflammatory infiltrate leukocyte count at the site of the lesion, in relation to the initial moment, there was effect of the experimental group, the time of analysis and interaction between these factors (twoway ANOVA test of repetitive measures, 0.001).

In the group analysis at different healing times, there was a gradual and significant increase in the amount of inflammatory infiltrate on 7 days of healing, in relation to 3 , 14 and 21 days (Tukey post-test, $p<0.05$ ).

In the comparison among the groups within each healing time, for the GPCvt.diab group, there was a significant increase in the amount of inflammatory infiltrate on the 3rd healing day, in relation to the other groups ( $p$ $<0.05$ ), being that on 21 days, the amount of leukocytes returned to similar values to those on 3 days $(p>0.05)$. 


\section{Total leukocyte count}

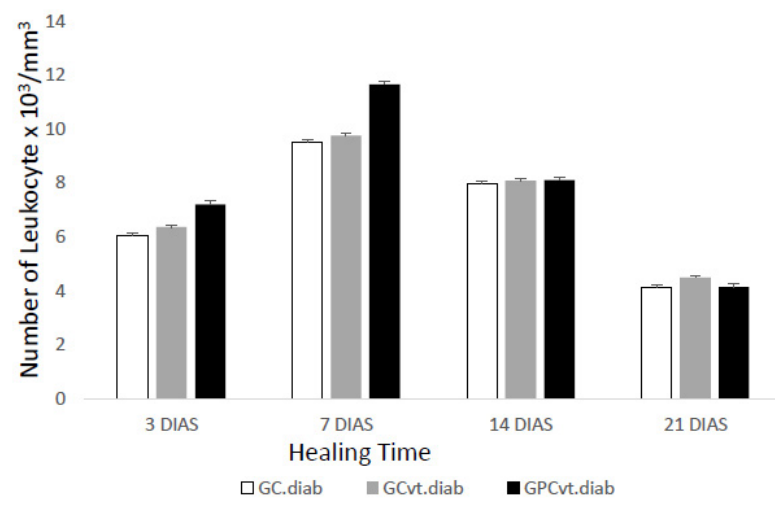

Figure 6 - Total peripheral blood leukocyte count (number of cells $/ \mathrm{mm} 3$ ) in each experimental group at analysis times 3, 7, 14 and 21 days. Data are presented as the mean \pm standard deviation of the mean (SEM) $(n=6)$. Diabetic Control Group (GC. diab), Topic Vehicle Control Group (GCvt.diab) and Experimental Group of powdered shell (GPCvt. diab).

In the general analysis of the results concerning the total leukocyte count in peripheral blood, in relation to the initial time, there was influence by the experimental group, the time of analysis and an interaction between these factors (two-way ANOVA test of repetitive measures, $p<0.001$ ) .

In the analysis by group in the different healing times, it was evidenced that there was a gradual and significant increase in leukocyte quantity at the moment 7 and 14 days, in relation to the moment 3 and 21 days (Tukey post-test, $p<0.05$ ), with no significant difference between moments 3 and 21 days ( $p>0.05$ ). In the groups GC.diab, GCvt.diab and GPCvt.diab there was a significant increase in the amount of leukocytes at the moments 3, 7 and 14 days, in relation to the moment 21 days $(p<0.05)$.

In the comparison between the experimental groups at each time of analysis, it was evidenced that the total amount of leukocytes in peripheral blood for the GPCvt. diab group on times 3 and 7 days was higher than the other groups ( $p<0.05)$, without significant difference between the groups GC.diab and GCvt.diab ( $p>0.05$ ), in these times.

\section{Discussion}

In this study the powdered shell of the $M$. lopesi snail was used as treatment of wounds in diabetic rats, since in a previous study the healing activity of the powdered shell was confirmed. The treatment efficacy of powdered shell healing wound is due to angiogenesis, initial proinflammatory activity and accelerated reduction of wound area ${ }^{5}$, which in diabetic animals are committed.

The process of wound healing occurs by mean of the synchronized interaction of cells, cytokines and growth factors ${ }^{7}$. Healing impairment is the most common complication associated with diabetes, with foot wounds occurring and mainly increasing the risk of limb amputation ${ }^{8}$. Healing can be interrupted at different stages in diabetes ${ }^{3}$, slowing the healing due to both intrinsic (hyperglycemia, neuropathy, angiopathy) and extrinsic causes (infections, callus formation and excessive pressure at the wound site due to and changed sensibility ${ }^{4}$.

The formation of new vessels in the wound site is a determining factor in the healing process either in diabetics or nondiabetics. Growth factors release and also other mediators stimulate the migration of endothelial cells from intact vessels within the wound margins to the wound area, thus promoting angiogenesis ${ }^{9-11,13}$.

In this study, the rates of inflammatory mediators were not quantified, but it is possible to infer that, in association with the increase in the total number of leukocytes in 
the peripheral blood and in the wound site, there was an increase in the mediator rate in the initial times of the healing process in the treated groups with the powdered shell of the snail, which, according to $\mathrm{NG}^{13}$, exacerbates the process of angiogenesis, being the vessels newly formed, responsible for maintaining the healing process, assisting in the production of collagen by fibroblasts and reepithelialization.

In the diabetic rat, the proinflammatory and angiogenesis activities are impaired, resulting in a slow healing process that in a quarter of cases is chronic. The natural compounds that favour the process of inflammation and angiogenesis during the wound healing process are of great importance for diabetic patients, since they are generally easily accessible and inexpensive ${ }^{14}$. The powdered shell of $M$. lopesi favoured angiogenesis on the 3rd and 7th day of the healing process in diabetic rats, which was also observed in non-diabetic rats ${ }^{5}$. However, on the 14th and 21st day of healing, the number of new formed vessels was not different in relation to the other groups. Angiogenesis has also been observed in wounds of diabetic rats with the administration of brown algae, Cystoseira trinodis $^{15}$, Curcumim ${ }^{16}$ and linoleic acid ${ }^{17}$, but these compounds showed angiogenic activity for more than 14 days.

Several factors interfere in angiogenesis process $^{18}$, but in diabetic rats, there is a marked expression of TGF- $\beta$, which at high levels impairs the formation of new vessels by the accentuated deposition of extracellular matrix ${ }^{8}$.

The less acid $\mathrm{pH}$ of wound site, associated with angiogenic activity at the initial healing times, favours the increase in the number of cells infiltrated at the wound site. The $\mathrm{pH}$ at the site of the wound during the acute phase is acid ( $\mathrm{pH}$ between 5 and 6$)$, by the accumulation of lactic acid. However, $\mathrm{CaCO}_{3}$ in aqueous solution suffers saline hydrolysis $\left(\mathrm{CaCO}_{3}+\mathrm{H}_{2} \mathrm{O} \rightarrow \mathrm{CO}_{2}+\mathrm{Ca}(\mathrm{OH})_{2}\right)$ producing a strong base, which would neutralize the increase of lactic acid ${ }^{19,20}$.

Cypraea moneta ${ }^{21}$, Megalobulimus lopesi $^{5}$, Haliotis diversicolor ${ }^{22}$, accelerated the healing process. The three species present in common, the mineral constitution of their shells, with $\mathrm{CaCO}_{3}$ being the main constituent mineral. Wong et $a .^{23}$ have observed that neutrophils from diabetic rats are more susceptible in expressing the neutrophil defense mechanism (NET) when stimulated with lithium-ionogenic solubilisin.

The Peptidyl Arginine Deiminase 4 (PAD4) calcium dependent enzyme, main responsible for mediating NET expression ${ }^{24}$, facilitates the chromatin decondensation, whereas in neutrophils of rats with type 2 diabetes can be found a higher concentration of serum calcium, which associated with the treatment with powdered shell of the snail would facilitate $\mathrm{NET}^{25}$.

With favourable $\mathrm{pH}$ and the number of new sufficient vessels, macrophages, fibroblasts and endothelial cells move into the wound space, filling it. Macrophages not only increase the inflammatory response but also secrete endothelial growth factor (VEGF) and fibroblast growth factor (FGF), promoting angiogenesis ${ }^{7}$. The powdered shell promoted an increase in the number of infiltrated cells at the wound site and formation of new vessels on days 3 and 7 of healing, which leads us to infer that there was synthesis and deposition of new extracellular matrix, improving the healing process in relation to the other groups.

Ram et al. ${ }^{26}$ observed early proinflammatory and late anti-inflammatory activity with the administration of bilirubin in wounds of diabetic rats, and the same effect 
was noticed with powder administration of $M$. lopesi shell. A healing effect was attributed to bilirubin, related to its ability to reduce oxidative stress in the wound of diabetic rats, accelerating the healing process. We infer that the same antioxidative effect may be present in the treatment with powdered shell, related to the formation of new vessels, increasing the blood supply to the wound site and thus reducing oxidative stress, since the depletion of ATP levels prevents cellular apoptosis, causing necrosis $^{7,21}$.

FGF, TGF- $\beta$ and platelet-derived growth factor (PDGF), cause fibroblast infiltration, and through TGF- $\beta$ and PDGF the phenotypic expression of fibroblasts is changed to myofibroblasts, which line up along the edges of the extracellular matrix generating constrictive force, that facilitates the closure of the wound ${ }^{7,21}$. The reduction in the area of the wound with the powdered shell was evident on the 21st day of healing, when compared to the other groups. This finding indicates acceleration in the process, with almost complete healing.

Chen et al. ${ }^{22}$ observed that with the treatment with the powdered shell of Haliotis diversicolor there was an increase in the phagocytic activity of macrophages, as well as an increase in the cellular pro-inflammatory activity in rats, accelerating the healing process. The results presented by Chen et al. ${ }^{22}$ are similar to those presented with $M$. lopesi powdered shell, and with 21 days of healing, both treatments presented total wound closure, but they obtained a better result than the control groups.

The extracellular calcium concentration is also associated with the cellular responses in the process of wound healing (influx of keratinocytes and fibroblasts) ${ }^{20}$. At the wound site, there is an increase in calcium concentration from the beginning of the healing process, persisting through the inflammatory, proliferative and remodelling phases. Zinc, copper, iron and magnesium present peak in the late inflammatory phase and in the phase of proliferation of epidermal and dermal cells, normalizing their levels during the remodelling phase $^{19,20}$.

The re-epithelialisation is another factor that assists in the closure of the wound, since they alter the keratinocyte phenotype of latent, migratory and proliferative, an action that is compromised in diabetic rats ${ }^{27}$. The powdered shell promoted an increase in the thickness of the new epithelium on the 21st day of healing, this finding reinforces the inferred for the reduction of the wound area, and the possible increase of TGF- $\beta 1$, associated with the reduction in the expression of TNF$\alpha$, favours re-epithelisation, contraction of the wound and consequent reduction of the wound. Another fact that reinforces this theory is that the powdered shell of Haliotis diversicolor promoted increased expression of TGF- $\beta 1$ during the healing process ${ }^{22}$.

The hypothesis of the increase of these mediators is strengthened when we analyze the evolution of the formation of new vessels, which on the 3rd and 7th day was higher in the treated group, being matched on the 14th and 21st day of healing. This effect may be due to the increase in mRNA expression for TGF- $\beta 1$, reducing angiogenic activity ${ }^{28}$ and accelerating the process of wound contraction and increase of new epithelium in diabetic rats ${ }^{27}$.

Another important event for the healing process is the inflammatory response. It is important to highlight the importance of the cytokine IL-10, produced after the injury by either immune cells or non-immune. IL-10 inhibits the production of proinflammatory cytokines by activated macrophages, as well as the infiltration of neutrophils and macrophages 
towards the lesion site ${ }^{29}$. Due to the increase in the total number of leukocytes in the peripheral blood and at the wound site, on the 3rd day of healing, it is suggested that the initial proinflammatory activity of the powdered shell of the snail is due to the inhibition of mRNA expression for IL -10 and/or which alters or destabilizes the cytokine structure, thus having marked initial proinflammatory activity and late anti-inflammatory activity.

Besides, the treatment with the powdered shell accelerated the healing process, as evidenced in the findings of the 21st day: progressive reduction in the number of inflammatory cells at the wound site, reduction of the wound area and increase of the thickness of the new epithelium. Unlike the results of Ram et al. ${ }^{26}$ that describe the antiinflammatory action as being favourable to angiogenesis, we did not show the same with the administration of the powdered shell of the snail, since the initial pro-inflammatory activity favoured the process healing in diabetic rats.

\section{Conclusions}

The effect of the mechanism of $M$. lopesi powdered shell on the wound healing process in diabetic rats by second intention, is related to the activation of macrophages in the initial stage of the healing process, having proinflammatory activity, as well as by the increase in the concentration of Calcium at the wound site, which assists in the maintenance of $\mathrm{pH}$, activates the NET and potentiates the cellular response in the process of wound healing (influx of keratinocytes and fibroblasts). Treatment with $M$. lopesi powdered shell in diabetic rats may have favoured healing by controlling the expression of cytokines and growth factors by recruiting cells that accentuate angiogenesis and wound closure, but future studies may to determine the activity in relation to the inflammatory mediators.

\section{References}

1. World Health Organization. World health statistics: a snapshot of global health. 2012.

2. Tfayli $H$, Silva A. Pathophysiology of type 2 diabetes mellitus in youth: the evolving chameleon. Arq Bras Endocrinol Metab. 2009;53(2):165-74. doi: 10.1590/S000427302009000200008.

3. Loots $M$, Kenter SB, van Galen WJ, Middelkoop E, Bos JD, Mekkes JR. Fibroblasts derived from chronic diabetic ulcers differ in their response to stimulation with EGF, IGF-I, FGF-b and PDGF compared to controls. Eur J Cell Biol. 2002;81:153-60. PMID: 11998867.

4. Jeffcoate W, Harding K. Diabetic foot ulcers. Lancet. 2003;361:1545-51. doi: 10.1016/ S0140-6736(03)13169-8.

5. Andrade PHM, Rondon SE, Carollo CA, Rodrigues Macedo ML, Viana LH, Schiaveto de Souza A, Turatti OC, Cepa Matos MF. Effect of powdered shells of the snail Megalobulimus lopesi on secondaryintention wound healing in an animal model. Evid Based Complementary Altern Med. 2015;2015:1-9. doi: 10.1155/2015/120785.

6. Marinho PC, Neto-Ferreira R, Carvalho JJ. Evaluation of therapeutic intervention with a natural product in cutaneous wound healing: the use of Capybara oil. Evid Based Complementary Altern Med. 2013;2013:110. doi: $10.1155 / 2013 / 217198$.

7. Kondo T, Ishida Y. Molecular pathology of wound healing. Forensic Sci Int. 2010;15(1-3):93-8. doi: 10.1016/j. forsciint.2010.07.004.

8. Costa PZ, Soares R. Neovascularization in diabetes and its complications. Unraveling the angiogenic paradox. Life Sci. 2013;92(22):1037-45. doi: 10.1016/j. Ifs.2013.04.001.

9. Fiedler U, Augustin HG. Angiopoietins: a link between angiogenesis and inflammation. Trends Immunol. 2006;27(12):552-8. PMID: 17045842 doi: 10.1016/j.it.2006.10.004.

10.Radziwon-Balicka A, Moncada CR, Zielnik $B$, Doroszko A, Jurasz P. Angiostatin inhibits endothelial MMP-2 and MMP-14 expression: a hypoxia specific mechanism of action. Vasc Pharmacol. 2013;58(4):280-91. 
doi: 10.1371/journal.pone.0059281.

11.Kubo H, Hayashi T, Ago K, Ago M, Kanekura T, Ogata M. Temporal expression of wound healingrelated genes in skin burn injury. Legal Med. 2014;16(1):8-13. doi: 10.1016/j. legalmed.2013.10.002.

12.Bahamman R, Mehrnoush $M$, Mohammad G, Aria M, Hamed M. Green tea and its antiangiogenesis effects. Biomed Pharmacother. 2017;89(2017): 949-56. doi: 10.1016/j. biopha.2017.01.161.

13.NG MF. The role of mast cells in wound healing. Int Wound J. 2010;7(1):55-61. doi: 10.1111/j.1742-481X.2009.00651.x.

14.Rodrigues HG, Vinolo MAR, Magdalon A, Vitzel K, Nachbar RT, Pessoa AFM, Santos MF, Hatanaka E, Calder PC, Curi $R$. Oral administration of oleic or linoleic acid accelerates the inflammatory phase of wound healing. J Investig Dermatol. 2012;132(1):208-15. doi: 10.1038/ jid.2011.265.

15.Madkour FF, Hassan MM, Abdo W, Khalil WF. Wound healing activity of brown algae plus polyherbal extract in normal and alloxan-induced diabetic rats. J Adv Vet Res. 2013;3(2013):102-8.

16.Kant V, Gopal A, Kumar D, Pathak NN, Ram M, Jangir BL, Tandan SK, Kumar D. Curcumin-induced angiogenesis hastens wound healing in diabetic rats. J Surg Res. 2015;193:978-88. doi: 10.1016/j. jss.2014.10.019.

17.Rodrigues HG, Vinolo MAR, Sato FT, Magdalon F, Kuhl CMC, Yamagata AS, Pessoa AFM, Malheiros G, Santos MF, Lima C, Farsky $\mathrm{SH}$, Camara NOS, Williner MR, Cury R. Oral administration of linoleic acid induces new vessel formation and improves skin wound healing in diabetic rats. PLoSONE. 2016;11(10):1-19. doi: 10.1371/journal. pone.0165115.

18.Bodnar RJ. Chemokine Regulation of angiogenesis during wound healing. Adv Wound Care. 2015;4(11):641-50. doi: 10.1089/wound.2014.0594.

19.Lansdown ABG. Calcium: a potential central regulator in wound healing in the skin. Wound Repair Regen. 2002;10:271-85. PMID: 12406163.

20.Limová P. Evaluation of two calcium alginate dressings in the management of venous ulcers. Europe PMC. 2003;49(9):26-33. PMID: 14581707.

21.Immanuel G, Berkmans JT, Muthusamy U, Ramasamy $R$, Santhiyagu $P$, Arunachalam P. Antipyretic, wound healing and antimicrobial activity of processed shell of the marine mollusc Cypraea moneta. Asian Pac J Trop Biomed. 2012;2012:1643-6. doi: 10.1016/s2221-1691(12)60469-9.

22.Chen ZC, Shing YSW, Wei YS, Yuan CL, Yi HL, Wei HW, Chun HC, Zhi HW. Anti-inflammatory and burn injury wound healing properties of the shell of Haliotis diversicolor. BMC Complement Altern Med. 2016;16:1-12. doi: 10.1186/s12906-016-1473-6.

23.Wong SL, Demers M, Martinod K, Gallant M, Yanming W, Goldfine AB, Kahn CR, Wagner DD. Diabetes primes neutrophils to undergo NETosis, which impairs wound healing. Nat Med. 2015;21(7):815-21. doi: 10.1038/ nm.3887.

24.Li $\mathrm{P}$, Ming $\mathrm{L}$, Michael $\mathrm{R}$, Lindberg $\mathrm{MJ}$, Kennett NX, Yanming W. PAD4 is essential for antibacterial innate immunity mediated by neutrophil extracellular traps. J Exp Med. 2010;207:1-10. doi: 10.1084/jem.20100239. 25.Leshner M, Hu W, Carrie L, Han Z, Xiangyun AC, Lorraine S, Yanming W. PAD4 mediated histone hypercitrullination induces heterochromatin decondensation and chromatin unfolding to form neutrophil extracellular trap-like structures. Front Immunol. 2012;3:1-11. doi: 10.3389/ fimmu.2012.00307.

26. Ram M Singh V, Kumawat S, Kant V, Tandan SK, Kumar D. Bilirubin modulated cytokines, growth factors and angiogenesis to improve cutaneous wound healing process in diabetic rats. Int Immunopharmacol. 2015;30(2016):137-49. doi: 10.1016/j. intimp.2015.11.037.

27. Galkowska H, Wojewodzka U, Olszeewski W. Chemokines, cytokines, and growth factors in keratinocytes and dermal endothelial cells in the margin of chronic diabetic foot ulcers. Wound Repair Regen. 2006;14(5):558-65. doi: 10.1111/j.1743-6109.2006.00155.x.

28.Geng L, Anathbandhu C, Geoffrey T, James LW, Jing W. TGF-beta suppresses VEGFAmediated angiogenesis in colon cancer metastasis. PLoSONE. 2013;8(3):1-17. doi: 10.1371/journal.pone.0059918. 
29.Goldberg MT, Yuan PH, Chunli Y, Michael CS, Warren LG. TNF-alpha suppresses alphasmooth muscle actin expression in human dermal fibroblasts: an implication for abnormal wound healing. J Investig Dermatol. 2007;127:2645-55. doi: 10.1038/ sj.jid.5700890.

\section{Correspondence:}

Paulo Henrique Muleta Andrade

Faculdade de Ciências Farmacêuticas,

Alimentos e Nutrição, UFMS

Cidade Universitária

79070-900 Campo Grande - MS Brasil

paulo.muleta@ufms.br

Received: Oct 16, 2017

Review: Dec 18, 2017

Accepted: Jan 19, 2018
Conflict of interest: none

Financial source: $\mathrm{CNPq}$
${ }^{1}$ Research performed at Department of Veterinary Medicine, Universidade Federal do Mato Grosso do Sul (UFMS), Campo GrandeMS, Brazil. 